\title{
Fixation of New Mutations in Small Populations
}

Michael C. Whitlock (whitlock@zoology.ubc.ca)

Reinhard Bürger (reinhard.buerger@univie.ac.at)

\section{Approved by}

Ulf Dieckmann (dieckman@iiasa.ac.at)

Program Leader, Adaptive Dynamics Network

November 2004 


\section{Contents}

1 Introduction 1

2 Purging and Fitness Changes in Declining Populations 1

3 Fixation of Deleterious Mutations: Mutational Meltdown 3

4 Factors Affecting Fixation of Deleterious Mutations 5

Effective population size and the Hill-Robertson effect . . . . . . . . . . . 5

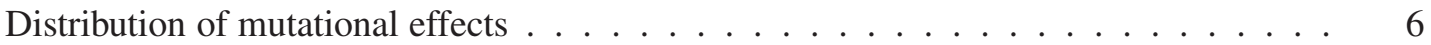

Dominance .............................. 7

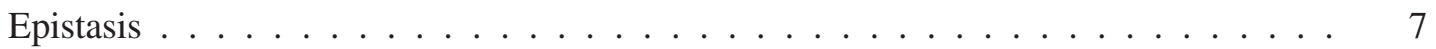

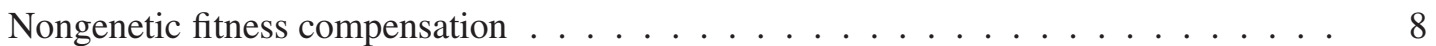

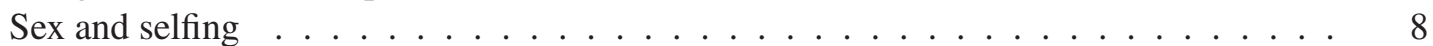

5 Fixation of Beneficial Mutations 9

Rate of back, beneficial, and compensatory mutations . . . . . . . . . . . . . 9

Rate of fixation of beneficial mutations in small populations _ . . . . . . . . . 10

Rate of fixation of mutations in declining populations . . . . . . . . . . . . . . 10

6 Time Scales for Extinction, Evolution, and Conservation 11

7 Concluding Comments $\quad 12$ 


\title{
About the Authors
}

\author{
Michael C. Whitlock \\ Department of Zoology \\ University of British Columbia \\ 6270 University Blvd. \\ Vancouver BC V6T 1Z4, Canada \\ Reinhard Bürger \\ Department of Mathematics \\ University of Vienna \\ Strudlhofgasse 4, A-1090 Vienna, Austria
}

\section{Acknowledgments}

We thank Lance Barrett-Lennard, Art Poon, Sally Otto, and Mike Lynch for their comments on this manuscript. M.C. Whitlock is supported by a grant from the Natural Science and Engineering Research Council of Canada. 


\title{
Fixation of New Mutations in Small Populations
}

\author{
Michael C. Whitlock \\ Reinhard Bürger
}

\section{Introduction}

Evolution proceeds as the result of a balance between a few basic processes: mutation, selection, migration, genetic drift, and recombination. Mutation is the ultimate source of all the genetic variation on which selection may act; it is therefore essential to evolution. Mutations carry a large cost, though; almost all are deleterious, reducing the fitness of the organisms in which they occur (see Chapter 7 in Ferrière et al. 2004). Mutation is therefore both a source of good and ill for a population (Lande 1995).

The overall effect of mutation on a population is strongly dependent on the population size. A large population has many new mutations in each generation, and therefore the probability is high that it will obtain new favorable mutations. This large population also has effective selection against the bad mutations that occur; deleterious mutations in a large population are kept at a low frequency within a balance between the forces of selection and those of mutation. A population with relatively fewer individuals, however, will have lower fitness on average, not only because fewer beneficial mutations arise, but also because deleterious mutations are more likely to reach high frequencies through random genetic drift. This shift in the balance between fixation of beneficial and deleterious mutations can result in a decline in the fitness of individuals in a small population and, ultimately, may lead to the extinction of that population. As such, a change in population size may determine the ultimate fate of a species affected by anthropogenic change.

This chapter reviews the genetic changes that occur as a result of a decrease in population size. We particularly focus on the fate of new mutations in a small population and the conditions under which a species may be at risk of extinction from harmful changes in fixation rates. We confine most of our comments to sexual, randomly mating populations.

\section{Purging and Fitness Changes in Declining Populations}

One of the unfortunate results of human activity in recent years is that the number of individuals in many species has declined rapidly and drastically, and this change seems likely to continue. This sudden change in population size can have several effects on the average fitness of a population.

Even a large population is expected to carry many copies of deleterious mutations, segregating at low frequencies at most of the loci in the genome. These rare alleles cause the mean fitness of the population to be lower than it would otherwise be; this reduction in fitness is called the mutation load (see Box 1). If the population's size becomes much lower than it was previously, there are a number of consequences. Many of the deleterious mutations already segregating in the population are lost immediately (or within a few generations) through sampling, but some may be fixed. Most of the deleterious mutations that persist in the population are, to some extent, recessive to the wildtype alleles, and a smaller population is more likely to express these alleles as homozygotes. Therefore, the strength of selection is increased proportionally, and the frequency of deleterious alleles becomes lower. This process is referred to as purging. While purging can reduce the mutation load for populations that are temporarily small [although not by much, Kirkpatrick 


\section{Box 1 Genetic load}

Genetic load is the term used to describe the reduction in fitness of a population, relative to some ideal population, due to the actions and interactions of various population genetic processes. The term load entered population genetics as a result of H.J. Muller's (1950) article Our load of mutations. Muller was concerned with the selective deaths required to remove new deleterious mutations from a population; we now refer to this type of load as mutation load, the reduction in fitness caused by segregating alleles brought into the population by mutation. An important result of Haldane (1937) is that if an infinitely large population is in a mutation-selection balance, then the mutation load $L$ is independent of the fitness effects of the mutations. More precisely, if $\mu$ denotes the total mutation rate from the wildtype (the allele with the highest fitness, normalized to 1) to all other possible alleles, then the mean fitness of the population $\bar{W}$ is

$$
\bar{W}=1-L=1-\mu,
$$

independent of the fitness values of the mutants (except for completely recessive alleles). This principle was extended to very general fitness and mutation patterns (Bürger and Hofbauer 1994; Bürger 2000, Chapter IV.5). The following theory assumes that each mutation occurs at a new locus, that loci are statistically independent (linkage equilibrium and no epistasis), and that every mutation reduces the fitness of its homozygous carrier by a factor $1-2 s$, where $s$ is the selection coefficient. The assumption that each mutation occurs at a new locus is adequate because mutation rates per locus are usually very low, so a mutation is lost or fixed before the next occurs. This, however, does not preclude the possibility of a high genome mutation rate $U$, because there are tens of thousands of genetic loci in genomes of larger organisms. Under the assumption that the actual number of mutations per individual is Poisson distributed with a mean $U$, one obtains

$$
\bar{W}=1-L \approx e^{-U} .
$$

In a finite population, the load $L_{\mathrm{S}}$ from segregating mutations approaches a stationary value with time and is close to zero in very small populations (less than 10-20 individuals) because of the reduced heterozygosity in such small populations. It increases to a maximum value at an intermediate population size [for genes with additive effects, this population size is approximately $1 /(2 s)$ ] and thereafter decreases to the infinite-population expectation, as $N_{e} s>5$, that is, $L_{\mathrm{S}} \approx 1-e^{-U}$ (see Kimura et al. 1963; Lynch et al. 1995a, 1995b). Here, $N_{e}$ denotes the so-called effective population size; it is explained in Section 4.

Alleles also sometimes fix through genetic drift. The resultant progressive reduction in mean fitness is sometimes referred to as drift load. Since, in a population of $N$ individuals, $U N$ mutations occur per generation, the expected fitness reduction caused by fixation in each generation is

$$
\Delta \bar{W}=\frac{\bar{W}^{\prime}}{\bar{W}}=(1-2 s)^{U N u_{f}},
$$

where $\bar{W}^{\prime}$ is the population's mean fitness in the next generation, $u_{f}$ is the fixation probability of a deleterious mutant (see Box 2). Mutation load is common to all populations, since all species have nonzero mutation rates for deleterious alleles. The drift load, on the other hand, is only important in relatively small populations.

Other types of load have been considered, such as the recombination load (a reduction in fitness that results from the breakup of fit gene combinations), the lag or evolutionary load (the deficit in fitness because of incomplete adaptation to a changing environment), and so on [see Crow and Kimura (1970) for a list]. We are not concerned with these kinds of load here (but see Chapter 10 in Ferrière et al. 2004). Finally, the concept of a load depends on the assumption of the existence of an optimal genotype or phenotype and, as such, is an idealization that has to be applied with caution.

and Jarne (2000)], in populations that are small for an extended period new mutations soon return the mutation load to a similar level as before the population size changed (see Box 1; Charlesworth et al. 1993a; Lynch et al. 1995a). Purging is not likely to increase the fitness of permanently small populations. 
In addition, and more importantly over a longer time scale, some of the mutations that occur at relatively low frequency in the large population drift to a higher frequency after the population has become smaller. If the new population is small enough, then some of these mutations fix, and the average fitness of the population is reduced as a result. The fitness reduction caused by the fixation of deleterious mutations through genetic drift is called drift load. If the population remains small, this process will continue with new mutations until the mean fitness of the population is sufficiently low that it cannot sustain itself. This decrease in fitness through the drift of deleterious mutations is thought to be the major genetic factor in determining the probability of extinction of a small population (Lande 1994; Lynch et al. 1995a, 1995b). The next sections deal with this issue in more detail.

\section{Fixation of Deleterious Mutations: Mutational Meltdown}

A species reduced to a small population size continues to have the same rate of mutation to inferior alleles, but if it is small enough some of these mutations increase in frequency through genetic drift until they replace the more fit allele. If a population has a reproductive rate $R_{0}$ (that is, each individual can produce $R_{0}$ offspring), then on average $1 / R_{0}$ of these offspring must survive to reproduce and keep the population from decreasing in size. As a population accumulates deleterious mutations, its intrinsic rate of increase becomes closer and closer to zero, until the mean fitness is below the point at which the population can survive. As the population size declines, deleterious alleles become more likely to fix and further reduce population size. This process has been called a mutational meltdown (Lynch and Gabriel 1990). This path to extinction will be fastest when:

- Deleterious mutations fix at a high rate;

- Fixed alleles have large effect; and

- The reproductive excess of the population is small.

The factors that accentuate these three terms are sometimes contradictory. As we show later, mutations of large effect are both rarer and less likely to fix. We consider each of these factors in turn.

The rate at which new harmful mutations are fixed depends on both the rate at which new mutants appear in the population and the probability that these new alleles fix. Empirical estimates suggest that the rate of deleterious mutations could be as high as one new mutation per gamete per generation, but this may be strongly species dependent (Crow and Simmons 1983; Eyre-Walker and Keightley 1999; Lynch et al. 1999; but see García-Dorado et al. 1999; also see Chapter 7 in Ferrière et al. 2004). Few of these mutations have very large effects on fitness; the vast majority have very small effects. On average, the homozygous effect of new mutations each generation is a few percent of fitness, maybe 2-15\% (Crow and Simmons 1983; Caballero and Keightley 1998; Lynch and Walsh 1998; Fry et al. 1999; Lynch et al. 1999; see also Chapter 7 in Ferrière et al. 2004). It is clear that without the counterbalance of effective selection, mutation would quickly erode the fitness of a population.

The ability of selection to keep deleterious alleles at a low frequency is diminished in small populations, because random genetic drift allows even a harmful allele to fix. The probability that a deleterious allele will fix is therefore a function of both its fitness effects and the effective size of the population. Box 2 summarizes the basic theory. Two main results are important. First, the probability of fixation is an exponentially decreasing function of the population size. For mutations of large effect, the effective population size must be very small to allow fixation (see Figure 1). Second, alleles with a selective effect that is less than about $1 /\left(2 N_{e}\right)$ are most likely to fix in the population. These alleles are called nearly neutral.

The effect on mean fitness of these fixed alleles is the product of the rate at which the alleles are fixed and their effects on fitness when homozygous. Alleles that have a very small effect are more likely to fix, but do not affect mean fitness much when they do. In contrast, alleles of large 
Box 2 Fixation of beneficial and deleterious alleles by genetic drift

When an allele appears initially in a population as a result of a mutation event, its frequency is $1 /(2 N)$, where $N$ is the census size of the population. For a new allele with fitness $1-s$ as a heterozygote and $1-2 s$ in the homozygous state, the probability $u_{f}$ that the allele will ultimately fix in the population was derived by Malecót (1952) and Kimura (1957), on the basis of a diffusion approximation, to be

$$
u_{f}=\frac{e^{2 s N_{e} / N}-1}{e^{4 s N_{e}}-1},
$$

which for $2 s N_{e} / N \ll 1$ is approximated well by

$$
u_{f}=\frac{2 s N_{e} / N}{e^{4 s N_{e}}-1} .
$$

The probability of fixation is therefore a function of the effective size of the population, the census size, and the strength of selection that acts on the allele. Bürger and Ewens (1995) showed that a better approximation is obtained by replacing $s$ in Equations (a) and (b) by $s /(1-s)$. Similar, but more complicated, equations that include the effects of dominance are available (see Crow and Kimura 1970).

Looking more carefully at Equation (a), we see that for beneficial alleles (in this notation given by $s<0)$, the probability of fixation is approximately $2|s| N_{e} / N$, if $|s|>1 /\left(2 N_{e}\right)$. Thus, even an allele with a strong favorable effect has a low probability of fixation. If the strength of selection is such that $|s|<1 /\left(2 N_{e}\right)$, the allele is said to be nearly neutral and the probability of fixation becomes almost independent of selection and approaches the neutral value, $u_{f}=1 /(2 N)$.

The probability of fixation of deleterious alleles decays nearly exponentially as the effective population size increases and becomes negligible if $s \gg 1 /\left(2 N_{e}\right)$. If, however, $s<1 /\left(2 N_{e}\right)$ the probability of fixation increases rapidly and approaches the neutral value of $1 /(2 N)$. In contrast to that of advantageous alleles, the fixation probability of a detrimental allele increases with decreasing effective population size (see Figure 1).

The decline of a population toward extinction begins when the mean absolute fitness drops below 1. Once this point is reached, population extinction occurs almost deterministically and very quickly. The mean extinction time of the population is determined mainly by the phase during which the mutations accumulate, mean fitness decreases, but the population size remains constant. The mutation load in the small population is approximately the same as that in the original larger population, so the mean fitness of the smaller population can be expressed as a function of its drift load and of its initial mean absolute fitness at low density, $\bar{W}_{0}$. If $T$ is the number of generations over which mutations accumulate by drift before the mean fitness becomes less than 1 , then

$$
\left(\Delta \bar{W}^{T} \bar{W}_{0}=1\right.
$$

where $\Delta \bar{W}$ is the change in mean fitness per generation and $\bar{W}_{0}$ is the average mean absolute fitness at low density.

Taking logarithms and substituting Equation (c) from Box 1 and Equation (b) from this box, the mean time in generations to extinction is given approximately by (Lande 1994; Lynch et al. 1995a)

$$
T \approx \frac{\left(e^{4 N_{e} s}-1\right) \ln \bar{W}_{0}}{4 U N_{e} s^{2}} .
$$

For mutations of variable effect, the expected decrease in fitness per generation at equilibrium is approximately

$$
\Delta \bar{W} \approx \int_{0}^{1 / 2} N U u_{f}(s) 2 s \Psi(s) d s,
$$




\section{Box 2 continued}

where $\Psi[s]$ is the probability that a new mutation has effect $s$ as a heterozygote. If we assume that the distribution of mutational effects is exponential with mean $\lambda$, the fixation flux as a result of drift can be found

$$
\Delta \bar{W} \approx \frac{U \zeta\left(3,1+\frac{1}{4 N_{e} \lambda}\right)}{8 N_{e}^{2} \lambda},
$$

where $\zeta$ is the generalized Riemann zeta function (Lande 1994). For cases in which the effective size is large enough such that the average mutant is not nearly neutral, the value of this $\zeta$ function is within $20 \%$ of 1 , and thus the decline in fitness with each generation in this model is approximately

$$
\Delta \bar{W} \approx \frac{U}{8 N_{e}^{2} \lambda} .
$$

From this it is obvious that as the population size increases, the rate of decline in fitness due to the fixation of deleterious mutations rapidly becomes small.

effect, if fixed, cause large changes in fitness, but this fixation is unlikely. It turns out that the largest effect on fitness results from mutations with mildly deleterious effects, with $s$ near $0.4 / N_{e}$ (Gabriel and Bürger 1994; Lande 1994); their fixation rate is relatively high but the effects on fitness are not negligible (see Figure 2).

If the population size is small enough that deleterious alleles accumulate, the mean fitness of the population slowly drops. Initially, this drop in fitness is relatively unimportant to the persistence of the population, because most species have reproductive excess, that is, much more reproductive capacity than required to replace dying members of the population. Eventually, though, as the less fit alleles accumulate, this excess decays and the population becomes incapable of sustaining itself, unless it is large enough to generate sufficient beneficial mutations. Therefore, whether a population eventually drops below this minimum fitness is a function of the mutation rate and population size, but how quickly this process leads to extinction is a function of reproductive excess as well. Populations with lower reproductive excess to begin with, as is often the case for much of the macrofauna beloved of conservation posters and polemics, are likely to be much more sensitive to genetic extinction than other species.

\section{Factors Affecting Fixation of Deleterious Mutations}

In this section we discuss several of the genetic and demographic properties of species that can substantially affect the basic process of mutation meltdown.

\section{Effective population size and the Hill-Robertson effect}

The effective population size, $N_{e}$, of a population is the size of an idealized population that has the same properties with respect to loss of variation by random drift as the population in question. As a rule, $N_{e}$ is smaller than the census size $N$ of the population, sometimes much smaller, and therefore genetic drift occurs at a faster rate than would be expected for $N$. The effective size is reduced by variance in reproductive success among individuals, including variance caused by unequal sex ratios, selection, and environmental effects. Populations are typically extremely variable in the reproductive success of their members; therefore $N_{e}$ is usually lower than $N$. The effective size is also decreased by variation over time in population size; the best description of the effects of drift in a population over time is given by the harmonic mean of the effective size of each generation. The harmonic mean of a set of highly variable numbers is usually much lower than the arithmetic 


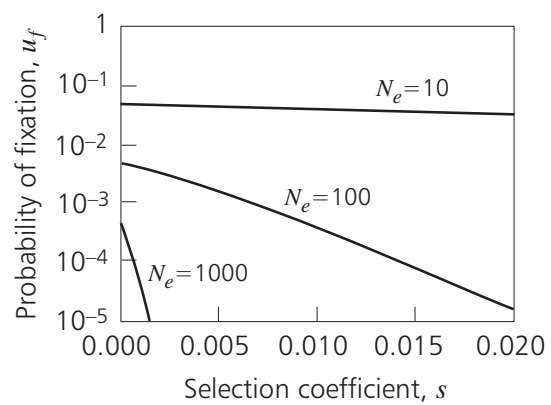

Figure 1 The probability of fixation of deleterious alleles depends on the strength of selection against them and the effective population size. The allele is initially present at frequency $1 /\left(2 N_{e}\right)$.

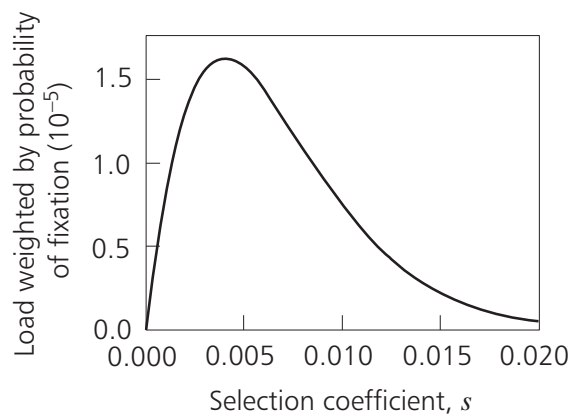

Figure 2 The total drift load attributable to an average new mutation is the product of the effect of that mutation when homozygous and the probability of fixation of that mutation. This load is maximized near a value of $s=0.4 / N_{e}$. Here, $N_{e}$ is set at 100 .

mean, so this form of averaging over time results in $N_{e}$ being much smaller than $N$. A recent review showed that when these factors are taken into account, or when $N_{e}$ is estimated indirectly, the ratio of $N_{e} / N$ is often as low as 0.1 or less (Frankham 1995c). Thus, populations are subject to much more genetic drift than their numbers alone may indicate.

One additional factor that influences the effective population size is genetic variance for fitness, such as that caused by segregating deleterious alleles. This not only results in variance in reproductive success, as mentioned in the previous paragraph, but also these alleles create correlations over the generations in which genotypes are successful. As a result, the variability in reproductive success is compounded over generations, and some alleles, independent of their own effects, may rise to high frequencies. This background selection (Charlesworth et al. 1993b) and hitchhiking (Maynard Smith and Haigh 1974) can result in a much smaller effective population size and so change the probability of fixation of other alleles (Hill and Robertson 1966). Simulations of the mutational meltdown process that include multiple loci showed that deleterious mutations accumulate much faster than expected by theory that does not account for the effects of background selection, particularly if the mutation rate is high and in relatively large populations (Lynch et al. 1995a).

\section{Distribution of mutational effects}

The probability of fixation of a deleterious mutation is a function of its selective effect. As mentioned above, the probability of fixation is greatest for small values of $s$, but the effect on mean fitness is maximized when $s$ is around $0.4 / N_{e}$ (see Figure 2). Thus, populations with many mutations that have selection coefficients around this value will decline in fitness rapidly relative to populations with the same number of mutations, but with larger or smaller effects.

For this reason, variance in mutational effects can make a large difference in the time to extinction. If the mean selection coefficient of a new mutation is much higher than $0.4 / N_{e}$, then variance in selective effect results in a faster decline of the population mean fitness (Lande 1994). 


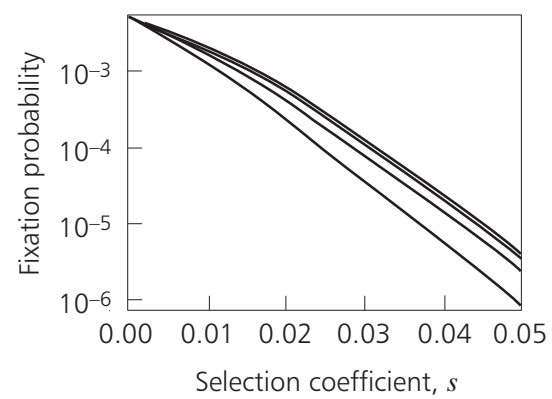

Figure 3 Deleterious recessive alleles are more likely to fix through drift than are additive alleles with the same homozygous effect, but this difference is not great. The five curves correspond (from top to bottom) to values of $h$ equal to $0,0.01,0.1,0.5$, and 1 , respectively. The curves for $h=0,0.01$, and 0.1 overlap almost completely on this graph. The fitnesses of the three genotypes are $1,1-2 h s$, and $1-2 s$, respectively. The effective population size is 100 .

This variance can result in orders of magnitude differences in the time to extinction. In contrast, if the mean mutation effect is close to this maximum effect size, then variance in mutational effect can only decrease the rate of loss of fitness (Lande 1994; Schultz and Lynch 1997). We have little direct evidence about the variance in mutational effects or, indeed, anything else about the shape of the distribution of the mutations, but it is clear that not all mutations have the same effect on fitness. Analysis by Keightley (1994) of Mukai's (1964) mutation accumulation data suggests that the distribution of mutational effects is extremely variable. Mackay et al. (1992b) showed that the distribution of the effects of the mutations caused by transposable element insertions is approximately exponential. Davies et al. (1999) show that many deleterious mutations have undetectably small effects.

\section{Dominance}

The quantitative conclusions above are based on alleles that interact additively with other alleles at the same locus. In fact, many deleterious alleles are recessive to their more fit counterparts. This recessivity increases the rate of fixation of deleterious mutations through drift (Kimura 1957; Crow and Kimura 1970; see Figure 3). The difference in the rates, though, is less than an order of magnitude. The reason for this increase in fixation rate is that the longer an allele segregates in a population at some frequency, the more chances there are that drift will fix that allele. Recessive alleles remain in populations longer than additive alleles with the same homozygous effect. Moreover, the probability of fixation is an increasing function of the allele frequency; recessive alleles are expected to have a much higher allele frequency than are additive alleles because of the mutation-selection balance. As a result of these two facts, the rate of fixation by drift of recessive alleles is somewhat greater than that of additively interacting ones. Indeed, some empirical analyses suggest that many deleterious mutations are nearly recessive (García-Dorado and Caballero 2001).

\section{Epistasis}

Not all alleles interact with alleles at other loci in an independent way, as assumed so far. If there is epistasis between loci, in particular if the deleterious effects of two loci combine to make an individual with a fitness worse than that predicted by the product of the fitness effects of the two loci, then the rate of fixation of deleterious mutations can be lower than predicted from single locus theory (Lande 1994; Schultz and Lynch 1997). This synergistic epistasis has been observed in Drosophila (Mukai 1964), but the evidence for an average level of epistasis being synergistic is weak. Other studies have found synergistic interaction in other species, but only among some pairs 
of loci and not others (Elena and Lenski 1997; de Visser et al. 1997; Whitlock and Bourguet 2000, reviewed in Phillips et al. 2000). Synergistic epistasis is unlikely to be very large on average, but even if it is large it will be unable to much change the time to extinction due to the fixation of deleterious mutations in sexual populations (see Butcher 1995).

\section{Nongenetic fitness compensation}

Several factors can contribute to deleterious alleles having more of an effect in large populations than in small. For example, if density affects the reproductive capacity of the species, as it often does, then the critical fitness to determine extinction rates is the fitness at low density, because populations must pass through a period of low density before extinction.

A more subtle factor is that the fixation rate of a deleterious allele is a function of the fitness of the allele relative to alternative alleles, while the drift load that results depends only on the absolute reduction in the number of surviving offspring per parent. Therefore, any mechanism that increases the differences in relative fitness of the alleles without increasing their effects on the absolute mean fitness will reduce the rate of the mutation meltdown. For example, an allele that reduces the competitive ability of males to attract mates can greatly reduce the relative fitness of an individual in a variable population and hence is much more unlikely to fix (Whitlock 2000). However, in a population fixed for this allele, the mean male mating success may be unaffected, as long as all females still mate. This allele would therefore be selected against and be less likely to fix through drift than if it did not affect male mating success, but once fixed this reduction in the relative mating success would have no effect on the productivity of the population as a whole. This effect is potentially large; there is a strong positive correlation between the effects of deleterious alleles on the productivity of a population and their effects on competitive mating success (Whitlock and Bourguet 2000). Consequently, the strength of selection against these alleles is much greater than would have been predicted from their effects on productivity alone, and they fix less often than would otherwise be expected. As a result of the nonlinear relationship between the strength of selection and probability of fixation, a small change in $s$ that does not result in a proportional change in mean fitness decline when fixed can reduce the effects of drift load by several orders of magnitude (Whitlock 2000).

\section{Sex and selfing}

So far only the effects of deleterious mutations in sexual, randomly mating populations have been discussed. The amount of drift load, and therefore the pace at which the mutational meltdown can occur, is expected to be much greater in either asexual populations or in populations that have a high degree of selfing. While these topics are too broad to give the detailed treatment they deserve in this chapter, we give the basic ideas of some of the results here.

The mutational meltdown process was first defined in terms of asexual populations (Lynch and Gabriel 1990). Without recombination between competing genotypes, deleterious alleles accumulate in asexual populations as a result of the stochastic loss of the fittest class with the fewest deleterious mutations. Without recombination, alleles are not required to fix in the population to reduce the evolutionary potential. If the class of genotypes with the fewest deleterious alleles is lost then it cannot be recreated by recombination, even if the fit versions of those alleles still exist in the population. This process is referred to as Muller's Ratchet because, as Muller described it, the population continually loses its fittest class, in which case the mean fitness can only decrease (Muller 1964; Felsenstein 1974; Charlesworth and Charlesworth 1997). The pace at which fitness is lost in an asexual population through new deleterious alleles is expected to be much faster than that in a sexual population of the same size. 
Selfing increases the pace of meltdown for similar reasons. Deleterious alleles are more likely to fix in selfing populations, because the effective population size tends to be smaller and because alleles that fix within a selfing lineage are less likely to recombine with genotypes from other lineages. As a result, the effectiveness of selection is diminished, and the population declines more rapidly in mean fitness (Pamilo et al. 1987; Charlesworth et al. 1993a; Schultz and Lynch 1997).

\section{Fixation of Beneficial Mutations}

The theory of mutational meltdown presented thus far assumes that all mutations are deleterious and that no mutations have a beneficial effect for the organisms that carry them. Of course, some mutations must be beneficial, or else evolution could not be a positive process and all species would become extinct. This section explores the effects of a small population size on the rate of incorporation of these beneficial mutations.

\section{Rate of back, beneficial, and compensatory mutations}

The justification for ignoring beneficial mutations is that beneficial mutations seem to be much rarer than deleterious ones, a fact borne out by mutation accumulation experiments (see Chapter 7 in Ferrière et al. 2004). However, many lines of evidence indicate that mutations with selective benefit do occur at nontrivial frequencies. Before this evidence is discussed, a few definitions are required. After a population has accumulated one or more deleterious mutations, it is possible for a new mutation to be beneficial even if it was not favored before. A new mutation can re-create a fit allele at a locus that had previously been fixed for a less fit allele; this is referred to as a back, or reverse, mutation. Furthermore it is possible that an allele at another locus, previously not favored by selection, may become selectively advantageous, perhaps because its action replaces or compensates for some of the effect of a deleterious mutant. These alleles are referred to as compensatory mutations. Finally, it is possible for a new allele to arise that is favored selectively in both the contexts of the deleterious allele and its absence; these mutations we include in the general group of beneficial mutants.

Back mutations for simple single-substitution mutations occur at a very low rate, and for some types of forward mutations, such as large deletions, back mutation is nearly impossible [see the references in Lande (1998b)]. However, back mutations probably represent only a small fraction of the mutations that are able to ameliorate the fitness loss of deleterious mutations. Biological systems are typically highly redundant, and changes in one part of that system can be compensated for by changes elsewhere. A particularly striking example of this was provided by an experiment with the RNA virus $\phi 6$ by Burch and Chao (1999). In this experiment, replicate populations of RNA viruses were fixed for a deleterious allele that arose spontaneously in their cultures. These populations were then kept at different population sizes, ranging from $N_{e}=60$ to 60000 , and the mean fitness of the populations was measured over time. In all but the smallest populations the fitness recovered to nearly the level of the nonmutant original strain. Moreover, this

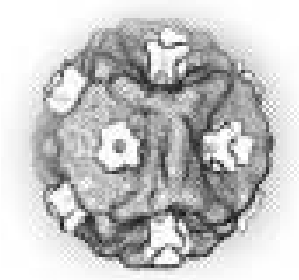

Phage $\phi 6$ recovery took place in a series of discrete steps, which implies that the evolution subsequent to the original deleterious mutation took place as a result of the fixation of new alleles at other loci, not merely by the fixation of a back mutation at this locus. As RNA viruses have much higher mutation rates than large eukaryotes, the rate of their recovery may be different to that expected for the class of organisms for which there is the most conservation concern; however, this experiment strongly indicates the potential for compensatory mutations that, if prevalent, can dramatically change the dynamics of meltdown to extinction, as shown below. 
Finally, a good geometric argument can be made that the number of beneficial mutations must increase as a population becomes more maladapted. Fisher (1930) argued that as a population neared its optimum, only mutations with a small effect could be beneficial, but if that same population were farther away from the optimum a much larger range of mutational effects would be adaptive. This argument could be advanced equally from the assumptions of quantitative genetics: if fixations of some alleles moved critical traits away from their fitness maxima, then any mutation that brings those traits back toward the optimum would be favored. A trait close to its best value would give a lower fitness if changed in value in either direction, while a trait away from its optimal value would be improved by that half of the possible changes that moved it back toward the optimum. By this line of argument, the possibility for compensatory mutations is large. The evolutionary consequences of genetic variability caused by such mutations with conditional fitness effects are explored in Chapter 10 in Ferrière et al. 2004.

\section{Rate of fixation of beneficial mutations in small populations}

The relative rarity of beneficial or compensatory mutations has caused some authors to ignore their effects when determining the rate of loss of fitness of a small population. In contrast, Lande (1998b) showed that the time to extinction can be changed by an order of magnitude or more if the effects of back mutations alone are included. Compensatory mutations would effectively increase the rate of back mutations and further slow or even halt the loss of fitness through drift [Poon and Otto 2000; Whitlock 2000; see also Wagner and Gabriel (1990) for a study of asexual populations]. While beneficial mutations may occur at a much lower rate than that for deleterious mutants, the selection process amplifies the fitness effects of these mutations because they have a relatively high probability of fixation.

There is, however, a critical population size at which the loss of fitness through the fixation of bad alleles cannot be compensated for by the fixation of favored alleles (Schultz and Lynch 1997). As the population size becomes small, the number of new favorable mutations that enters a population becomes low, because it is proportional to the population size. Simultaneously, the probability of fixation of beneficial mutations decreases as the effective population size decreases, while that of deleterious alleles increases. For small values of $N_{e}$, both classes have nearly the same fixation probability, namely that of neutral mutations (see Box 2). As a result there is a critical effective size at which the effects of the fixation of beneficial mutations cannot balance the fixation of deleterious mutations, and the population begins to lose mean fitness. This balance point is likely reached with effective population sizes in the low hundreds and depends on the mean effect of mutations and the proportion of mutations that are beneficial (Whitlock 2000).

\section{Rate of fixation of mutations in declining populations}

The paragraphs above deal with the effects of a constant small population size on the probability of fixation of new mutations. In reality, threatened populations may have a population size that is declining over time because of external factors. In such cases, the probability of fixation of new alleles with selective advantage $|s|$ becomes $2(|s|+r) N_{e} / N$, where $r$ is the growth rate of the population size per generation and $r<0$ in declining populations (Otto and Whitlock 1997). For values of $r$ just below zero, this can nearly negate the probability of fixation of beneficial alleles. For negative values of $(|s|+r)$, there is essentially no possibility that the beneficial allele will be fixed.

Similarly, the probability of fixation of deleterious alleles in a declining population is greatly increased. In populations that are steadily reducing in size, the probability of fixation of an allele is given by the standard equation [Equation (a) in Box 2], but with the fixation effective population size given by $N_{e} \approx N(1+r /|s|)$ (Otto and Whitlock 1997). If the rate of population decline is a 


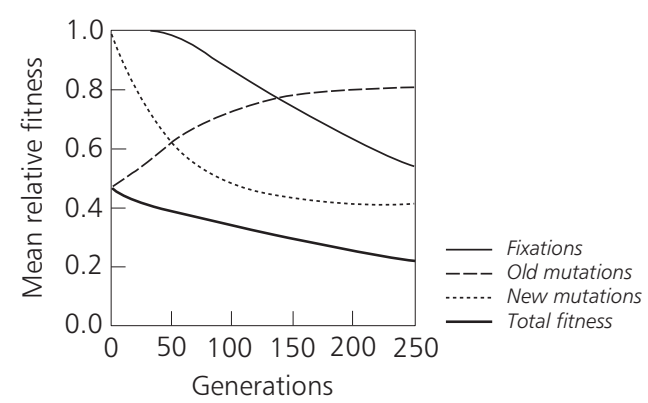

Figure 4 The evolution of mean fitness in a finite monoecious population of effective size $N_{e}=32$, the ancestral population of which was infinite and in mutation-selection balance. The genomic mutation rate is $U=0.75$, and the heterozygous effect of each deleterious mutation is $s=0.025$, which gives an initial mean fitness of $e^{-U}=0.472$. The fitness from "old" mutations includes purging and the fixation of deleterious alleles in the founder individuals, whereas the curve for "new" mutations refers only to segregating genes. The curve "Fixations" represents the fitness that results from the fixation of new and old deleterious mutations. Source: Lynch et al. (1995a).

substantial fraction of the selection coefficient, then the effective size can be drastically reduced by population decline.

\section{Time Scales for Extinction, Evolution, and Conservation}

The most important question about this topic from a conservation biology perspective is, "How fast will a population of this size go extinct?" This question has been addressed by a number of authors, and the answer is, "It depends." We can, however, make some strong qualitative statements.

When a population is reduced in size artificially, many of the deleterious mutations carried by it before the population decline are lost through genetic drift or purging, but some become fixed through genetic drift. The population continues to have new mutations, most of which are quickly lost, some of which persist for long enough to affect the population by mutation load, and a few of which are eventually fixed in the population through drift. It is this last phase of the process that presents the most danger to the population; the continued fixation of deleterious mutations may eventually result in a loss of fitness of the population such that it cannot sustain itself and it declines to extinction (see Figure 4). The critical effective population size at which this occurs depends on mutation rates and on assumptions about the possible effects the mutations might have.

The earliest estimates of the potential for drift load to cause extinction of a sexual population suggested that this was only likely to occur with a population size less than 100 (Charlesworth et al. 1993a). This study used a fixed mutational effect with only deleterious alleles, but more importantly it assumed that each individual was capable of producing an unlimited number of offspring. Hence, the population was unlikely to ever go extinct. Subsequent studies that relaxed this last assumption and set a limit on the reproductive capacity of individuals found that populations with effective size in the hundreds could be expected to become extinct within a few hundred or thousand generations (Lande 1994; Lynch et al. 1995a, 1995b; see Figure 5). These population sizes are effective sizes and so are likely to be an order of magnitude lower than the census size, which implies that for populations to be sustainable over more than a few hundred generations they must number in the thousands or tens of thousands.

Several factors make these predictions approximate. We have few data on the distribution of mutational effects; there is even great uncertainty about the mutation rate itself. It may be that mutational effects on the order of $0.4 / N_{e}$ are uncommon, which could substantially prolong the expected lifespan of a species (Lande 1994). Furthermore, mutations of beneficial effect are likely to be non-negligible and reduce the risk of extinction substantially (Lande 1998b; Whitlock 


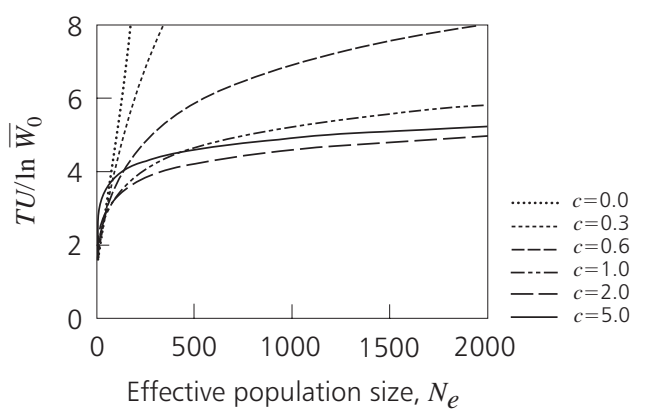

Figure 5 Length $T$ of the phase of mutation accumulation until mean fitness is less than 1 [Equation (c) in Box 2] as a function of the effective population size $N_{e} ; T$ is scaled by the genomic mutation rate $U$ and the logarithm of the initial mean fitness $\bar{W}_{0}$. It is assumed that the distribution of mutational effects $\Psi(s)$ is a gamma distribution with a mean of 0.025 and a coefficient of variation of $c$. The choice $c=1$ yields an exponential distribution. For large values of $c$, the distribution is highly kurtotic (e.g., with $c=5$, the kurtosis is 150). Source: Lande (1998b).

2000). On the other hand, these models do not account for demographic stochastic effects, which could substantially increase the risk of extinction of an already mutation-weakened population. The calculations typically assume additive allelic effects, which somewhat underestimates the probability of fixation and therefore the rate of fitness loss. Finally, a small population size may substantially impair the ability to respond to environmental change (see Chapter 10 in Ferrière et al. 2004). With this uncertainty, prudence argues for policies that err on the side of caution.

Unfortunately, these ideas are difficult to test experimentally. The declines in mean fitness expected through the meltdown are likely to take tens or hundreds of generations and should not show much effect on population productivity in the short term. It is essential that we learn more about the distribution of mutational effects and that careful long-term experiments be carried out to test the outcomes of these models (see Chapter 5 in Ferrière et al. 2004).

\section{Concluding Comments}

If a species' population size was reduced to the hundreds, would it persist long enough for these genetic considerations to matter? To some extent this is not known, although current theory suggests that for populations of this size the risk presented by mutational meltdown is greater than the threat of demographic stochasticity and on a par with the risks of environmental stochasticity (Lande 1994). Of course, the risk of extinction caused by these longer term factors is irrelevant if sufficient habitat and protection is not secured for the short term.

The key parameters in determining whether a population will go extinct for genetic reasons and how long this will take are the effective population size, the mutation rate, and the distribution of deleterious and beneficial mutational effects. The parameter that we may possibly control is the effective population size, which is related to the census population size, which in turn is related to the amount of undisturbed habitat and protection a species receives. It behooves us to use that control to maintain sufficiently large populations to prevent any meltdown to extinction.

\section{References}

Burch CL \& Chao L (1999). Evolution by small steps and rugged landscapes in the RNA virus $\phi 6$. Genetics 151:921-927

Bürger R (2000). The Mathematical Theory of Selection, Recombination, and Mutation. Chichester, UK: Wiley \& Sons 
Bürger R \& Ewens WJ (1995). Fixation probabilities of additive alleles in diploid populations. Journal of Mathematical Biology 33:557-575

Bürger R \& Hofbauer J (1994). Mutation load and mutation-selection balance in quantitative genetic traits. Journal of Mathematical Biology 32:193-218

Butcher D (1995). Muller's ratchet, epistasis and mutation effects. Genetics 141:431-437

Caballero A \& Keightley PD (1998). Inferences on genome-wide deleterious mutation rates in inbred populations of Drosophila and mice. Genetica 102/103:229-239

Charlesworth B \& Charlesworth D (1997). Rapid fixation of deleterious alleles can be caused by Muller's ratchet. Genetical Research 67:27-41

Charlesworth D, Morgan MT \& Charlesworth B (1993a). Mutation accumulation in finite outbreeding and inbreeding populations. Genetical Research 61:39-56

Charlesworth D, Morgan MT \& Charlesworth B (1993b). The effect of deleterious mutations on neutral molecular variation. Genetics 134:1289-1303

Crow JF \& Kimura M (1970). An Introduction to Population Genetics Theory. New York, NY, USA: Harper \& Row

Crow JF \& Simmons MJ (1983). The mutation load in Drosophila. In The Genetics and Biology of Drosophila, Vol. 3c, eds. Ashburner M, Carson HL \& Thompson JN Jr, pp. 1-35. New York, NY, USA: Academic Press

Davies EK, Peters AD \& Keightley PD (1999). High frequency of cryptic deleterious mutations in Caenorhabditis elegans. Science 285:1748-1751

De Visser JA, Hoekstra RF \& van den Ende H (1997). An experimental test for synergistic epistasis and its application in Chlamydomonas. Genetics 145:815-819

Elena SF \& Lenski RE (1997). Test of synergistic interactions among deleterious mutations in bacteria. Nature 390:395-398

Eyre-Walker A \& Keightley PD (1999). High genomic deleterious mutation rates in hominids. Nature 397:344-347

Felsenstein J (1974). The evolutionary advantage of recombination. Genetics 78:737-756

Ferrière R, Dieckmann U \& Couvet D (2004). Evolutionary Conservation Biology. Cambridge, UK: Cambridge University Press.

Fisher RA (1930). The Genetical Theory of Natural Selection. Oxford, UK: Oxford University Press

Frankham R (1995c). Effective population size/adult population size ratios in wildlife: A review. Genetical Research 66:95-107.

Fry JD, Keightley PD, Heinsohn SL \& Nuzhdin SV (1999). New estimates of the rates and effects of mildly deleterious mutation in Drosophila melanogaster. Proceedings of the National Academy of Sciences of the USA 96:574-579

Gabriel W \& Bürger R (1994). Extinction risk by mutational meltdown: Synergistic effects between population regulation and genetic drift. In Conservation Genetics, eds. Loeschcke V, Tomiuk J \& Jain SK, pp. 70-84. Basel, Switzerland: Birkhäuser

Garciía-Dorado A \& Caballero A (2001). On the average coefficient of dominance of deleterious spontaneous mutations. Genetics 155:1991-2001

Garciía-Dorado A, López-Fanjul C \& Caballero A (1999). Properties of spontaneous mutations affecting quantitative traits. Genetical Research 74:341-350

Haldane JBS (1937). The effect of variation on fitness. The American Naturalist 71:337-349

Hill WG \& Robertson A (1966). The effect of linkage on limits to artificial selection. Genetical Research 8:269-294

Keightley P (1994). The distribution of mutation effects on viability in Drosophila melanogaster. Genetics 138:1315-1322

Kimura M (1957). Some problems of stochastic processes in genetics. Annals of Mathematical Statistics 28:882-901 
Kimura M, Maruyama T \& Crow JF (1963). The mutation load in small populations. Genetics 48:1303-1312

Kirkpatrick M \& Jarne P (2000). The effects of a bottleneck on inbreeding depression and the genetic load. The American Naturalist 155:154-167

Lande R (1994). Risk of population extinction from fixation of new deleterious mutations. Evolution 48:1460-1469

Lande R (1995). Mutation and conservation. Conservation Biology 9:782-791

Lande R (1998b). Risk of population extinction from fixation of deleterious and reverse mutations. Genetica 102/103:21-27

Lynch M \& Gabriel W (1990). Mutation load and survival of small populations. Evolution 44:1725-1737

Lynch M \& Walsh B (1998). Genetics and Analysis of Quantitative Traits. Sunderland, MA, USA: Sinauer Associates Inc.

Lynch M, Conery J \& Bürger R (1995a). Mutation accumulation and the extinction of small populations. The American Naturalist 146:489-518

Lynch M, Conery J \& Bürger R (1995b). Mutational meltdown in sexual populations. Evolution 49:1067-1080

Lynch M, Blanchard J, Houle D, Kibota T, Schultz S, Vassilieva L \& Willis J (1999). Perspective: Spontaneous deleterious mutation. Evolution 53:645-663

Mackay TFC, Lyman RF \& Jackson MS (1992b). Effects of P-element insertions on quantitative traits in Drosophila melanogaster. Genetics 130:315-332

Malecót G (1952). Les processes stochastiques et la méthode des fonctions génératrices ou caracteréstiques. Publications de l'Institut de Statistique de l'Université de Paris 1: 3:1-16

Maynard Smith J \& Haigh J (1974). The hitchhiking effect of a favorable gene. Genetical Research 23:23-35

Mukai T (1964). The genetic structure of natural populations of Drosophila melanogaster I. Spontaneous mutation rate of polygenes controlling viability. Genetics 50:1-19

Muller HJ (1950). Our load of mutations. American Journal of Human Genetics 2:111-176

Muller HJ (1964). The relation of recombination to mutational advance. Mutation Research 1:2-9

Otto SP \& Whitlock MC (1997). Fixation of beneficial mutations in a population of changing size. Genetics 146:723-733

Pamilo P, Nei M \& Li W-H (1987). Accumulation of mutations in sexual and asexual populations. Genetical Research 49:135-146

Phillips PC, Otto SP \& Whitlock MC (2000). Beyond the average: The evolutionary importance of epistasis and the variability of epistatic effects. In Epistasis and the Evolutionary Process, eds. Wolf J, Brodie III ED \& Wade MJ, pp. 20-38. Oxford, UK: Oxford Press

Poon A \& Otto SP (2000). Compensating for our load of mutations: Freezing the meltdown of small populations. Evolution 54:1467-1479

Schultz ST \& Lynch M (1997). Mutation and extinction: The role of variable mutational effects, synergistic epistasis, beneficial mutations, and degree of outcrossing. Evolution 51:13631371

Wagner GP \& Gabriel W (1990). Quantitative variation in finite parthenogenetic populations: What stops Muller's ratchet in the absence of recombination? Evolution 44:715-731

Whitlock MC (2000). Fixation of new alleles and the extinction of small populations: Drift load, beneficial alleles, and sexual selection. Evolution 54:1855-1861

Whitlock MC \& Bourguet D (2000). Factors affecting the genetic load in Drosophila: Synergistic epistasis and correlations among fitness components. Evolution 54:1654-1660 